\title{
Efficacy of pulsed radiofrequency in controlling pain caused by spinal disorders: a narrative review
}

\author{
Seoyon Yang ${ }^{1}$, Min Cheol Chang ${ }^{2}$ \\ ${ }^{1}$ Department of Rehabilitation Medicine, Ewha Woman's University Seoul Hospital, Ewha Woman's University School of Medicine, Seoul, Korea; \\ ${ }^{2}$ Department of Rehabilitation Medicine, College of Medicine, Yeungnam University, Daegu, Republic of Korea \\ Contributions: (I) Conception and design: All authors; (II) Administrative support: All authors; (III) Provision of study materials or patients: All \\ authors; (IV) Collection and assembly of data: All authors; (V) Data analysis and interpretation: All authors; (VI) Manuscript writing: All authors; (VII) \\ Final approval of manuscript: All authors. \\ Correspondence to: Min Cheol Chang. Department of Physical Medicine and Rehabilitation, College of Medicine, Yeungnam University 317-1, \\ Daemyungdong, Namku, Daegu 705-717, Republic of Korea. Email: wheel633@ynu.ac.kr.
}

\begin{abstract}
Pulsed radiofrequency (PRF) stimulation has been safely and effectively applied for controlling various types of pain. Here, we aimed to systematically review the literature pertaining to the efficacy of PRF stimulation for managing pain associated with spinal disorders. We conducted a PubMed search for papers published until August 20, 2019, that used PRF to treat pain resulting from spinal disorders. The following inclusion criteria were applied when selecting the articles: (I) patients' pain was caused by spinal disorders; (II) PRF stimulation was applied on the spinal structure; and (III) after PRF stimulation, follow-up evaluation was performed to assess the change in pain intensity. Review articles were excluded. The primary literature search yielded 168 relevant papers. After reading their titles and abstracts and evaluating their eligibility based on the full-text articles, we finally included 59 publications in this review. The therapeutic outcomes reported in the selected studies showed that PRF is an effective treatment for cervical and lumbar radicular pain. Similarly, PRF stimulation seems to be effective for treating cervical joint (cervical facet and atlantoaxial joints) pain and lumbar facet joint pain. PRF stimulation has also resulted in positive outcomes in some studies, in which patients were treated for other disorders, including cervicogenic headache, discogenic neck pain, thoracic facet joint pain, discogenic back pain, and coccydynia. Nevertheless, there is insufficient evidence for the efficacy of PRF stimulation in these disorders. In conclusion, our review provides insights into the degree of evidence available on the effectiveness of PRF stimulation for treating pain associated with each of the spinal disorders reviewed. This information will help clinicians make informed decisions on using PRF stimulation to treat various spinal conditions and manage the associated pain.
\end{abstract}

Keywords: Cervicogenic headache; coccydynia; discogenic pain; joint pain; pulsed radiofrequency (PRF); radicular pain; spinal disorder.

Submitted Jan 31, 2020. Accepted for publication Jul 31, 2020.

doi: 10.21037/apm-20-298

View this article at: http://dx.doi.org/10.21037/apm-20-298

\section{Introduction}

Pain caused by spinal disorders is common in the human population, resulting in functional impairment and decreased quality of life (1). Spinal pain includes axial pain and radicular pain. Axial pain originates mainly from the disc and the facet joints. Radicular pain is caused by irritation of the sensory root or the dorsal root ganglion (DRG) of a spinal nerve. Several therapeutic methods, including oral medications, modalities, and corticosteroid injections, are being used to treat spinal pain (1). However, in some patients, the pain does not respond to these treatment methods. Moreover, oral pain-relief medication can cause adverse effects in the gastrointestinal, renal, or 
cardiovascular systems. Corticosteroid injections have several adverse effects as well, including tissue atrophy, fat necrosis, degeneration of the articular cartilage, hyperglycemia, hematoma, vascular necrosis, and injection (1).

Pulsed radiofrequency (PRF) stimulation can safely and effectively control various types of pain (2-6). Continuous radiofrequency $(\mathrm{CRF})$ stimulation exposes the target nerves to continuous electrical stimulation, which subsequently increases the temperature around the radiofrequency needle tip, causing a lesion in the target nerve and ablates the surrounding structures. In contrast, PRF stimulation uses radiofrequency currents to produce heat bursts, with long resting phases between them; therefore, the tissue temperature does not increase beyond $42^{\circ} \mathrm{C}$, which prevents irreversible tissue damage (2-6). The exact mechanism of pain reduction by PRF stimulation has not yet been fully elucidated, but some possible mechanisms have been proposed. PRF stimulation inhibits the propagation of pain impulses by decreasing microglial activity and increasing c-fos expression in the dorsal horn. Together, these sustain the activation of pain-inhibitory mechanisms $(7,8)$. Moreover, downregulation of microglia after PRF stimulation was reported. Because microglia contribute to the development of chronic pain by releasing several cytokines that mediate the pain signal, downregulating the microglia might prevent the progression to chronic pain (7). Additionally, PRF stimulation causes microscopic damage to the principal sensory nociceptive sensory fibers (C-fibers and A-delta fibers), but rarely damages the larger nonpain-related sensory fibers (A-beta fibers) (9). Finally, PRF stimulation was reported to enhance the noradrenergic and serotonergic descending pain inhibitory pathways (2).

Herein, we review published studies to establish the effectiveness of PRF stimulation in managing pain caused by spinal disorders.

\section{Methods}

We searched the MEDLINE database (PubMed) for articles published until August 20, 2019, by using the following key phrases: (PRF AND spine) OR (PRF AND radicular pain) OR (PRF AND radiculopathy) OR (PRF AND facet joint) OR (PRF AND discogenic pain) OR (PRF AND atlanto-occipital joint) OR (PRF AND atlanto-axial joint). Articles meeting the following inclusion criteria were selected: (I) patients' pain was caused by spinal disorders; (II) PRF stimulation was applied on the spinal structure; (III) after PRF stimulation, follow-up evaluation was performed to assess the change in pain intensity. Review articles were excluded.

\section{Results}

The primary literature search yielded 168 relevant papers. After reading their titles and abstracts, and assessing their eligibility based on the full-text articles, we included 58 publications in this review. Among the included studies, 22 reported applying PRF stimulation for cervical spine disorders [radicular pain, 13 (10-22); joint pain, six (3,23-27); discogenic pain, one (28); cervicogenic headache, two $(29,30)]$. PRF stimulation was also applied for lumbar spine disorders in 37 studies [radicular pain, 23 (11,15,16,31-50); joint pain, ten (24,51-59); discogenic pain, four (60-63)]. Pain due to thoracic spine disorders was treated with PRF stimulation in one study (64), and that due to coccydynia was treated with PRF stimulation in three studies (65-67).

\section{Discussion}

\section{Cervical spine disorders}

Pain caused by cervical spine disorders includes radicular pain, joint (facet, atlanto-occipital, and atlanto-axial) pain, discogenic pain, and cervicogenic headache.

\section{Cervical radicular pain}

The leading causes of radicular pain are herniated disc or spinal stenosis that induce chemical inflammation and mechanical compression of the nerve root (68). Thirteen studies reported using PRF stimulation for cervical radicular pain; all studies conducted PRF stimulation of the cervical dorsal root ganglion (DRG) and showed a positive pain-reduction response (10-22). Four of these studies were randomized controlled trials (RCTs) $(14,15,18,20)$. Van Zundert et al. (18) showed better treatment outcomes in the 11 patients who received PRF stimulation treatment than in the 12 patients who received sham treatment. The assessment was done three months after the treatment. Lee et al. (15) also performed a 3-month follow-up and reported similarly positive treatment response in the ten patients treated with PRF stimulation and in the eight patients who received transforaminal epidural steroid injection (TFESI). Wang et al. (20) reported better treatment outcomes with combination therapy of PRF stimulation and TFESI than with either of them alone. Halim et al. (14) compared the pain-reduction effect between PRF 
stimulation and percutaneous nucleoplasty in 34 patients. Both procedures successfully reduced cervical radicular pain and had sustained effect for at least three months, with no significant intergroup differences. These RCTs show that PRF stimulation could be used for managing cervical radicular pain, and that the addition of TFESI to the treatment could enhance its effect. Among the other nine studies, three were prospective observational studies that showed a significant decrease in the initial pain $(12,13,17)$. Choi et al. reported a sustained PRF stimulation effect for one year (12). In their study, 14 of 21 patients showed pain relief of $\geq 50 \%$ when assessed one year after the application of PRF stimulation to the cervical DRG. Four retrospective studies $(11,16,21,22)$ and two case reports $(10,19)$ also showed that PRF stimulation could effectively control cervical radicular pain. Interestingly, Chang conducted bipolar PRF stimulation in two patients whose pain could not be controlled by combination therapy of monopolar PRF stimulation and TFESI (10). Bipolar PRF stimulation successfully controlled the pain, with the effects lasting at least six months. These favorable outcomes indicate that PRF stimulation is a good therapeutic option for managing cervical radicular pain.

\section{Cervical joint pain}

Six studies reported performing PRF stimulation to control pain in the cervical joints, including the atlanto-occipital and cervical facet joints (3,23-37). PRF stimulation was performed using medial branch stimulation $(23,24)$ or intra-articular stimulation (3,25-27). PRF stimulation of the medial branch of the cervical posterior primary ramus was successful in inhibiting pain signal transmission from the facet joint to the brain $(23,24)$. In intra-articular PRF stimulation, owing to the insulating property of the bone, the current produced by the PRF stimulation was deflected by bony surfaces and thus remained within the joint space, without any reduction in intensity $(3,25-27)$. The current in the joint space could thus inhibit the excitement of the nociceptive nerve fibers present in the synovial lining of the joint (3,25-27). Lim et al. (3) performed intra-articular PRF stimulation in 20 patients and intra-articular corticosteroid injection in 20 patients. Both treatments performed equally well at reducing the patients' pain when assessed one, three, and six months after treatment. Shin et al. (25) conducted intra-articular PRF stimulation (12 patients) or intra-articular corticosteroid injection (11 patients) to treat atlanto-occipital joint pain. Both procedures showed positive effects in reducing the atlanto-occipital joint pain, and their effects persisted for at least six months. No significant intergroup differences were reported. Moreover, Liliang et al. (23) conducted a prospective observational study to evaluate the effect of PRF stimulation of the cervical medial branch on neck pain due to whiplash in 14 patients. At the 1-year follow-up, nine patients (64.3\%) showed significant pain reduction. Two retrospective studies and one case report $(24,26,27)$ showed substantial pain reduction after a medial branch or intra-articular PRF stimulation. These outcomes suggest that PRF stimulation is effective in patients with cervical joint pain. Despite these favorable treatment outcomes, additional prospective clinical trials would be required to ascertain the usefulness of PRF stimulation in the treatment of cervical joint pain.

\section{Cervicogenic headache and discogenic neck pain}

PRF stimulation of the C2 DRG is used for managing cervicogenic headaches. The C2 DRG is a common clinical target for various types of headache treatment (69). The medial branch of the $\mathrm{C} 2$ spinal nerve dorsal ramus becomes the greater occipital nerve, which is the primary sensory nerve of the skull occipital area (69). Considering the convergence in the trigeminal nucleus in the upper cervical segments, inhibiting the transmission of nociceptive information in the C2 DRG by PRF stimulation should be able to resolve cervicogenic headache (69). Zhang et al. (30) reported of two patients whose cervicogenic headache was successfully resolved after PRF stimulation of the C2 DRG. Li et al. (29) found that, when treating patients with cervicogenic headache, interlaminar epidural steroid injection, combined with PRF stimulation of the C2 DRG, had a better effect than did treatment with epidural steroid injection alone.

Additionally, Kwak et al. (28) performed C4-C5 intradiscal PRF stimulation in a patient with a discogenic neck pain that scored 7 on a numeric rating scale (NRS). Two weeks and one month after the PRF stimulation, the patient had no pain at all, and two months after the PRF stimulation, the NRS score was 2.

Although the aforementioned studies imply that PRF stimulation is useful in treating these conditions, more definitive evidence from trials assessing PRF stimulation as a treatment for cervicogenic headache and discogenic neck pain is needed.

\section{Thoracic spine disorder}

Only one study reported of pain caused by a thoracic 
spine disorder that was treated with PRF stimulation (64). Chang (64) performed a prospective study, using PRF stimulation of the thoracic medial branch of the dorsal ramus in 20 patients with chronic thoracic facet joint pain that was refractory to medial branch block with local anesthetics. Three months after the PRF stimulation, 11 patients $(55 \%)$ showed $\geq 50 \%$ reduction in their pain score, with the average NRS score going down from 6 before the treatment to 4 three months after the treatment. The extreme scarcity of PRF stimulation studies for treating thoracic spine pain is probably because of the relatively low incidence rate of thoracic spine disorders. Moreover, the diagnostic difficulty might have partly contributed to the lack of studies on the effectiveness of PRF stimulation in treating pain originating in the thoracic spine. Further studies are, therefore, essential.

\section{Lumbosacral spine disorders}

The most common forms of pain associated with lumbosacral spine disorders are radicular, facet joint, and discogenic pain.

\section{Lumbosacral radicular pain}

To date, 23 studies have investigated the effects of PRF stimulation of the DRG in patients with lumbosacral radicular pain induced by herniated discs, spinal stenosis, or failed back surgery syndrome $(11,15,16,31-50)$. Of these studies, five were RCTs $(15,33,36,42,43)$, six were prospective observational studies, ten were retrospective studies, and two were case reports. Among the RCTs, Simopoulos et al. (43) evaluated the effect of combined CRF and PRF stimulation treatment. Both the PRF stimulation group (37 patients) and the combined treatment group (39 patients) showed a good pain-reduction effect with no difference between the groups. However, the addition of CRF stimulation tended to lengthen the average duration of PRF stimulation analgesic effect from 3.2 to 4.4 months. Shanthanna et al. (42) reported relatively small, but better, pain-reduction effect in 14 patients who received the PRF stimulation to the DRG, when compared to the 15 patients who received a placebo intervention. Koh et al. (36) found that combination treatment with PRF stimulation and TFESI had better outcomes than did TFESI alone when assessed two and three months after treatment. Lee et al. (15) reported that both PRF stimulation of the DRG (9 patients) and TFESI (11 patients) showed significant pain reduction at 2, 4, 8, and 12 weeks after treatment, with similar degrees of pain relief in both treatment groups. Chang et al. (33) compared the effects of monopolar and bipolar PRF stimulation of the DRG in 40 patients and showed that bipolar PRF stimulation was more effective than monopolar PRF stimulation in controlling lumbosacral radicular pain.

Six prospective observational studies also showed that PRF stimulation could effectively manage lumbosacral radicular pain $(37,39,45,46,49,50)$. Lee et al. (37) performed bipolar PRF stimulation of the DRG in 23 patients whose radicular pain did not respond to the combined treatment of monopolar PRF stimulation and TFESI. The average NRS score changed from 6 to 3.4 at three months after the treatment with bipolar PRF stimulation. Twelve patients $(52.2 \%)$ reported $\geq 50 \%$ pain reduction.

Ten retrospective studies $(11,16,31,35,38,40,41,44,47,48)$ and two case reports $(24,32)$ have all found that PRF stimulation had a positive effect on lumbosacral pain. Abejón et al. (31) reported that radicular pain due to a herniated lumbar disc or spinal stenosis was well-controlled by PRF stimulation. However, patients with failed back surgery syndrome responded poorly to the treatment with PRF stimulation of the DRG. Park and Lee (41) reviewed the treatment outcome of 82 patients with failed back surgery syndrome and found that PRF stimulation of the DRG was only weakly effective in reducing the lumbosacral radicular pain in these patients.

Almost all studies on PRF stimulation for lumbosacral radicular pain showed its usefulness in controlling this pain. The exceptions to this rule are the study by Shanthanna et al. (42) and patients with failed back surgery syndrome in the study by Abejón et al. (31). Therefore, it can be concluded that PRF stimulation could be beneficial in alleviating lumbosacral radicular pain. However, welldesigned RCTs with a sham or a placebo group are warranted to confirm the usefulness of PRF stimulation of the DRG for such pain relief. Additionally, future studies should categorize the cervical spine disorders and adjust for factors affecting the outcomes of the treatment with PRF stimulation.

\section{Lumbosacral facet joint pain}

Among the ten studies on lumbosacral facet joint pain (24,51-59), four were RCTs. Tekin et al. (59) compared the effects of PRF and CRF stimulation of the lumbar medial branch in 60 patients by dividing them into three equal-sized groups: control, PRF stimulation, and CRF stimulation. Immediately after each procedure, the PRF and CRF stimulation groups showed more pain reduction 
than did the control group. However, at 6 and 12 months after the treatment procedures, the CRF stimulation group showed a sustained pain-reduction effect, but the PRF stimulation group did not. This study, however, is limited in that the first follow-up was only 6 months after treatment; hence, the effects of PRF stimulation could not have been adequately evaluated. Kroll et al. (56) performed PRF or CRF stimulation in two groups of 13 patients each, and found that PRF stimulation of the lumbar medial branches was ineffective in controlling lumbosacral facet joint pain. In contrast, CRF stimulation showed a significant pain reduction at the 3 -month posttreatment evaluation. However, their study is limited by the small sample size. Hashemi et al. (55) compared the therapeutic effect of PRF stimulation of the lumbar medial branch to that of intra-facet joint corticosteroid injection in 80 patients with degenerative spondylolisthesis. At the 6-week post-treatment evaluation, the effects of both treatments were similar. However, at the 3-and 6-month post-treatment evaluations, the PRF stimulation group showed better pain reduction and functional improvement. Do et al. (54) performed an RCT on 60 patients, comparing intra-articular PRF stimulation with intra-facet joint corticosteroid injection. Both procedures successfully controlled facet joint pain, and their positive effect persisted for at least 6 months. At 1 month after the treatment, the corticosteroid injection group showed greater pain reduction. Yet, at 3 and 6 months after the treatment, there were no differences between the groups. Among the four abovementioned RCTs (54-56,59), one study reported about positive therapeutic effects of PRF stimulation of the lumbar medial branches (55) and another when the stimulation was done in the intra-facet joint (54). The other two studies $(56,59)$ did not find any positive effect of PRF stimulation when treating lumbar facet joint pain. However, considering the limitations of these two studies, their utility in determining the effectiveness of PRF stimulation in treating lumbar facet joint pain remains questionable.

Despite the inconsistent results of these four RCTs (54-56,59), the other six studies on a medial branch and intra-articular PRF stimulation [two prospective observational studies $(52,53)$, three retrospective studies $(24,51,57)$, and one case report (58)] showed positive pain reduction and functional improvement during short- or long-term follow-up.

Although the limited number of available studies is insufficient to confirm the effectiveness of PRF stimulation in treating lumbosacral facet joint pain, these studies demonstrate that medial branch or intra-articular PRF stimulation is useful for managing such pain.

\section{Discogenic lower back pain}

Discogenic lower back pain is a common form of lower back pain (61). Its primary etiology is abnormal nerve ingrowth and nociceptive pain in the outer annulus fibrosus (61). Although many minimally invasive intradiscal procedures, such as intradiscal electrothermal therapy and CRF ablation, are available, the treatment is still challenging (61). The possibility of using PRF stimulation treatment for discogenic lower back pain has been investigated. PRF stimulation could inhibit the transfer of pain signals from the abnormally growing intradiscal nociceptive nerves. To date, four prospective observational studies (60-63) have reported successful outcomes following intradiscal PRF stimulation treatment, with pain being significantly lower even after one year (60-63).

Despite the positive outcomes in these studies, more RCTs should be performed to support a broader application of PRF stimulation treatment for discogenic back pain. Shortening of the intervertebral disc as an adverse effect of inserting the PRF catheter should also be investigated, for example, by using follow-up magnetic resonance imaging.

\section{Coccydynia}

Coccydynia refers to pain in and around the coccygeal region (65-67). Procedures for managing coccydynia include caudal epidural corticosteroid injection, a corticosteroid injection into the intercoccygeal joint, and ganglion impar block with an anesthetic agent and corticosteroids (70). One case report (66) and one retrospective study (67) reported that PRF stimulation of the ganglion impar reduced coccydynia. Gopal et al. (66) retrospectively reviewed the data of 20 patients who received PRF stimulation of the ganglion impar to treat coccydynia. Fifteen patients (75\%) showed positive treatment effects. Atim et al. (65) retrospectively studied the effect of caudal epidural PRF stimulation in 21 patients with coccydynia. Their median NRS score had reduced from 8 before treatment to 2 at the 3-week and 6-month follow-ups.

Nevertheless, no prospective study has investigated the effect of PRF stimulation when used to treat coccydynia, possibly because of its low prevalence. Therefore, further studies are warranted to confirm the therapeutic effectiveness of PRF stimulation for coccydynia. 


\section{Conclusions}

This review shows that PRF stimulation treatment could be beneficial in controlling pain caused by spinal disorders. None of the 58 reviewed studies reported any serious complications. Moreover, the compelling evidence supports PRF stimulation as an effective treatment for cervical and lumbar radicular and facet joint pain. Nevertheless, to promote a broader application of PRF stimulation as a treatment modality in these spinal disorders, we need more well-designed RCTs that would support the positive therapeutic effects of PRF stimulation. Evidence on the effectiveness of PRF stimulation treatment for pain arising from other spinal disorders, including cervicogenic headache, discogenic neck pain, thoracic facet joint pain, discogenic back pain, and coccydynia, is still lacking, even though some studies have reported positive therapeutic effects. Further well-designed studies are warranted to clarify the application of PRF stimulation treatment for these types of pain disorders. Moreover, studies that would compare the effect of PRF stimulation and analgesic medication have not been conducted. Therefore, researches on this topic are still needed. This is the first review to assess the effectiveness of PRF stimulation with a focus on treating spinal pain. Our review provides insights into the degree of available evidence for each of the spinal pain disorders. This information could help clinicians make informed decisions when considering the use of PRF stimulation for the treatment of various spinal pain conditions.

\section{Acknowledgments}

Funding: This work was supported by the 2020 Yeungnam University Research Grant.

\section{Footnote}

Conflicts of Interest: Both authors have completed the ICMJE uniform disclosure form (available at http://dx.doi. org/10.21037/apm-20-298). The authors have no conflicts of interest to declare.

Ethical Statement: The authors are accountable for all aspects of the work in ensuring that questions related to the accuracy or integrity of any part of the work are appropriately investigated and resolved.

Open Access Statement: This is an Open Access article distributed in accordance with the Creative Commons Attribution-NonCommercial-NoDerivs 4.0 International License (CC BY-NC-ND 4.0), which permits the noncommercial replication and distribution of the article with the strict proviso that no changes or edits are made and the original work is properly cited (including links to both the formal publication through the relevant DOI and the license). See: https://creativecommons.org/licenses/by-nc-nd/4.0/.

\section{References}

1. Galan-Martin MA, Montero-Cuadrado F, Lluch-Girbes E, et al. Pain Neuroscience Education and Physical Therapeutic Exercise for Patients with Chronic Spinal Pain in Spanish Physiotherapy Primary Care: A Pragmatic Randomized Controlled Trial. J Clin Med 2020;9:E1201.

2. Cho IT, Cho YW, Kwak SG, et al. Comparison between ultrasound-guided interfascial pulsed radiofrequency and ultrasound-guided interfascial block with local anesthetic in myofascial pain syndrome of trapezius muscle. Medicine (Baltimore) 2017;96:e6019.

3. Lim JW, Cho YW, Lee DG, et al. Comparison of Intraarticular Pulsed Radiofrequency and Intraarticular Corticosteroid Injection for Management of Cervical Facet Joint Pain. Pain Physician 2017;20:E961-7.

4. Park SM, Sho YW, Ahn SH, et al. Comparison of the effects of ultrasound-guided interfascial pulsed radiofrequency and ultrasound-guided interfascial injection on myofascial pain syndrome of the gastrocnemius. Ann Rehabil Med 2016;40;885-92.

5. Ye L, Mei Q, Li M, et al. A comparative efficacy evaluation of ultrasound-guided pulsed radiofrequency treatment in the gastrocnemius in managing plantar heel pain: a randomized and controlled trial. Pain Med 2015;16:782-90.

6. Zhao WX, Wang Q, He MW, et al. Radiofreuqency thermocoagulation combined with pulsed radiofrequency helps relieve postoperative complications of trigeminal neuralgia. Genet Mol Res 2015;14:7617-23.

7. Cho HK, Cho YW, Kim EH, et al. Changes in pain behavior and glial activation in the spinal dorsal horn after pulsed radiofrequency current administration to the dorsal root ganglion in a rat model of lumbar disc herniation: Laboratory investigation. J Neurosurg Spine 2013;19:256-63.

8. Higuchi Y, Nashold BS Jr, Sluijter M, et al. Exposure of the dorsal root ganglion in rats to pulsed radiofrequency currents activates dorsal horn lamina I and II neurons. 
Neurosurgery 2002;50:850-5; discussion 856.

9. Erdine S, Bilir A, Cosman ER, et al. Ultrastructural changes in axons following exposure to pulsed radiofrequency fields. Pain Pract 2009;9:407-17.

10. Chang MC. Effect of bipolar pulsed radiofrequency on refractory chronic cervical radicular pain: A report of two cases. Medicine (Baltimore) 2017;96:e6604

11. Chao SC, Lee HT, Kao TH, et al. Percutaneous pulsed radiofrequency in the treatment of cervical and lumbar radicular pain. Surg Neurol 2008;70:59-65; discussion 65 .

12. Choi GS, Ahn SH, Cho YW, et al. Long-term effect of pulsed radiofrequency on chronic cervical radicular pain refractory to repeated transforaminal epidural steroid injections. Pain Med 2012;13:368-75.

13. Choi GS, Ahn SH, Cho YW, et al. Short-term effects of pulsed radiofrequency on chronic refractory cervical radicular pain. Ann Rehabil Med 2011;35:826-32.

14. Halim W, van der Weegen W, Lim T, et al. Percutaneous Cervical Nucleoplasty vs. Pulsed Radio Frequency of the Dorsal Root Ganglion in Patients with Contained Cervical Disk Herniation; A Prospective, Randomized Controlled Trial. Pain Pract 2017;17:729-37.

15. Lee DG, Ahn SH, Lee J. Comparative Effectivenesses of Pulsed Radiofrequency and Transforaminal Steroid Injection for Radicular Pain due to Disc Herniation: a Prospective Randomized Trial. J Korean Med Sci 2016;31:1324-30.

16. Shabat S, Pevsner Y, Folman Y, et al. Pulsed radiofrequency in the treatment of patients with chronic neuropathic spinal pain. Minim Invasive Neurosurg 2006;49:147-9.

17. Van Zundert J, Lamé IE, de Louw A, et al. Percutaneous pulsed radiofrequency treatment of the cervical dorsal root ganglion in the treatment of chronic cervical pain syndromes: a clinical audit. Neuromodulation 2003;6:6-14.

18. Van Zundert J, Patijn J, Kessels A, et al. Pulsed radiofrequency adjacent to the cervical dorsal root ganglion in chronic cervical radicular pain: a double blind sham controlled randomized clinical trial. Pain 2007;127:173-82.

19. Vigneri S, Sindaco G, Zanella $M$, et al. Interventional treatment for neuropathic pain due to combined cervical radiculopathy and carpal tunnel syndrome: a case report. Clin Case Rep 2017;5:414-8.

20. Wang F, Zhou Q, Xiao L, et al. A Randomized Comparative Study of Pulsed Radiofrequency Treatment With or Without Selective Nerve Root Block for Chronic Cervical Radicular Pain. Pain Pract 2017;17:589-95.
21. Xiao L, Li J, Li D, et al. A posterior approach to cervical nerve root block and pulsed radiofrequency treatment for cervical radicular pain: a retrospective study. J Clin Anesth 2015;27:486-91.

22. Yoon YM, Han SR, Lee SJ, et al. The efficacy of pulsed radiofrequency treatment of cervical radicular pain patients. Korean J Spine 2014;11:109-12.

23. Liliang PC, Lu K, Hsieh CH, et al. Pulsed radiofrequency of cervical medial branches for treatment of whiplashrelated cervical zygapophysial joint pain. Surg Neurol 2008;70 Suppl 1:S1:50-5; discussion S1:55.

24. Mikeladze G, Espinal R, Finnegan R, et al. Pulsed radiofrequency application in treatment of chronic zygapophyseal joint pain. Spine J 2003;3:360-2.

25. Shin SM, Kwak SG, Lee DG, et al. Clinical Effectiveness of Intra-articular Pulsed Radiofrequency Compared to Intra-articular Corticosteroid Injection for Management of Atlanto-occipital Joint Pain: A Prospective Randomized Controlled Pilot Study. Spine (Phila Pa 1976) 2018;43:741-6.

26. Sluijter ME, Teixeira A, Serra V, et al. Intra-articular application of pulsed radiofrequency for arthrogenic pain-report of six cases. Pain Pract 2008;8:57-61.

27. Tak HJ, Chang MC. Effect of Pulsed Radiofrequency Therapy on Chronic Refractory Atlanto-Occipital Joint Pain. World Neurosurg 2018;119:e638-42.

28. Kwak SY, Chang MC. Effect of intradiscal pulsed radiofrequency on refractory chronic discogenic neck pain: A case report. Medicine (Baltimore) 2018;97:e0509.

29. Li SJ, Feng D. Pulsed radiofrequency of the C2 dorsal root ganglion and epidural steroid injections for cervicogenic headache. Neurol Sci 2019;40:1173-81.

30. Zhang J, Shi DS, Wang R. Pulsed radiofrequency of the second cervical ganglion $(\mathrm{C} 2)$ for the treatment of cervicogenic headache. J Headache Pain 2011;12:569-71.

31. Abejón D, Garcia-del-Valle S, Fuentes ML, et al. Pulsed radiofrequency in lumbar radicular pain: clinical effects in various etiological groups. Pain Pract 2007;7:21-6.

32. Abejón D, Ortego R, Solís R, et al. Trans-facet-joint approach to pulsed radiofrequency ablation of the L5 dorsal root ganglion in a patient with degenerative spondylosis and scoliosis. Pain Pract 2008;8:202-5.

33. Chang MC, Cho YW, Ahn SH. Comparison between bipolar pulsed radiofrequency and monopolar pulsed radiofrequency in chronic lumbosacral radicular pain: A randomized controlled trial. Medicine (Baltimore) 2017;96:e6236.

34. Hussain AM, Afshan G. Use of pulsed radiofrequency in 
failed back surgery syndrome. J Coll Physicians Surg Pak 2007;17:353-5.

35. Kim SJ, Park SJ, Yoon DM, et al. Predictors of the analgesic efficacy of pulsed radiofrequency treatment in patients with chronic lumbosacral radicular pain: a retrospective observational study. J Pain Res 2018;11:1223-30.

36. Koh W, Choi SS, Karm MH, et al. Treatment of chronic lumbosacral radicular pain using adjuvant pulsed radiofrequency: a randomized controlled study. Pain Med 2015;16:432-41.

37. Lee DG, Cho YW, Ahn SH, et al. The Effect of Bipolar Pulsed Radiofrequency Treatment on Chronic Lumbosacral Radicular Pain Refractory to Monopolar Pulsed Radiofrequency Treatment. Pain Physician 2018;21:E97-103.

38. Martin DC, Willis ML, Mullinax LA, et al. Pulsed radiofrequency application in the treatment of chronic pain. Pain Pract 2007;7:31-5.

39. Mehta V, Snidvongs S, Ghai B, et al. Characterization of peripheral and central sensitization after dorsal root ganglion intervention in patients with unilateral lumbosacral radicular pain: a prospective pilot study. Br J Anaesth 2017;118:924-31

40. Nagda JV, Davis CW, Bajwa ZH, et al. Retrospective review of the efficacy and safety of repeated pulsed and continuous radiofrequency lesioning of the dorsal root ganglion/segmental nerve for lumbar radicular pain. Pain Physician 2011;14:371-6.

41. Park CH, Lee SH. The Outcome of Pulsed Radiofrequency Treatment According to Electodiagnosis in Patients with Intractable Lumbosacral Radicular Pain. Pain Med 2019;20:1697-701.

42. Shanthanna H, Chan P, McChesney J, et al. Pulsed radiofrequency treatment of the lumbar dorsal root ganglion in patients with chronic lumbar radicular pain: a randomized, placebo-controlled pilot study. J Pain Res 2014;7:47-55.

43. Simopoulos TT, Kraemer J, Nagda JV, et al. Response to pulsed and continuous radiofrequency lesioning of the dorsal root ganglion and segmental nerves in patients with chronic lumbar radicular pain. Pain Physician 2008;11:137-44.

44. Teixeira A, Grandinson M, Sluijter ME. Pulsed radiofrequency for radicular pain due to a herniated intervertebral disc--an initial report. Pain Pract 2005;5:111-5.

45. Tsou HK, Chao SC, Wang CJ, et al. Percutaneous pulsed radiofrequency applied to the L-2 dorsal root ganglion for treatment of chronic low-back pain: 3-year experience. J Neurosurg Spine 2010;12:190-6.

46. Van Boxem K, de Meij N, Kessels A, et al. Pulsed radiofrequency for chronic intractable lumbosacral radicular pain: a six-month cohort study. Pain Med 2015;16:1155-62.

47. Van Boxem K, de Meij N, Patijn J, et al. Predictive Factors for Successful Outcome of Pulsed Radiofrequency Treatment in Patients with Intractable Lumbosacral Radicular Pain. Pain Med 2016;17:1233-40.

48. Van Boxem K, van Bilsen J, de Meij N, et al. Pulsed radiofrequency treatment adjacent to the lumbar dorsal root ganglion for the management of lumbosacral radicular syndrome: a clinical audit. Pain Med 2011;12:1322-30.

49. Vigneri S, Sindaco G, Gallo G, et al. Effectiveness of pulsed radiofrequency with multifunctional epidural electrode in chronic lumbosacral radicular pain with neuropathic features. Pain Physician 2014;17:477-86.

50. Yang CL, Yang BD, Lin ML, et al. A patient-mount navigated intervention system for spinal diseases and its clinical trial on percutaneous pulsed radiofrequency stimulation of dorsal root ganglion. Spine (Phila $\mathrm{Pa}$ 1976) 2010;35:E1126-32.

51. Chang MC, Cho YW, Ahn DH, et al. Intraarticular Pulsed Radiofrequency to Treat Refractory Lumbar Facet Joint Pain in Patients with Low Back Pain. World Neurosurg 2018;112:e140-4.

52. Çetin A, Yektaş A. Evaluation of the Short- and LongTerm Effectiveness of Pulsed Radiofrequency and Conventional Radiofrequency Performed for Medial Branch Block in Patients with Lumbar Facet Joint Pain. Pain Res Manag 2018;2018:7492753.

53. Colini-Baldeschi G. Evaluation of pulsed radiofrequency denervation in the treatment of chronic facetjoint pain: an observational study. Anesth Pain Med 2012;1:168-73.

54. Do KH, Ahn SH, Cho YW, et al. Comparison of intraarticular lumbar facet joint pulsed radiofrequency and intra-articular lumbar facet joint corticosteroid injection for management of lumbar facet joint pain: A randomized controlled trial. Medicine (Baltimore) 2017;96:e6524.

55. Hashemi M, Hashemian M, Mohajerani SA, et al. Effect of pulsed radiofrequency in treatment of facet-joint origin back pain in patients with degenerative spondylolisthesis. Eur Spine J 2014;23:1927-32.

56. Kroll HR, Kim D, Danic MJ, et al. A randomized, double-blind, prospective study comparing the efficacy of continuous versus pulsed radiofrequency in the treatment 
of lumbar facet syndrome. J Clin Anesth 2008;20:534-7.

57. Lindner R, Sluijter ME, Schleinzer W. Pulsed radiofrequency treatment of the lumbar medial branch for facet pain: a retrospective analysis. Pain Med 2006;7:435-9.

58. Schianchi PM. A new technique to treat facet joint pain with pulsed radiofrequency. Anesth Pain Med 2015;5:e21061.

59. Tekin I, Mirzai H, Ok G, Erbuyun K, et al. A comparison of conventional and pulsed radiofrequency denervation in the treatment of chronic facet joint pain. Clin J Pain 2007;23:524-9.

60. Fukui S, Nitta K, Iwashita N, et al. Intradiscal pulsed radiofrequency for chronic lumbar discogenic low back pain: a one year prospective outcome study using discoblock for diagnosis. Pain Physician 2013;16:E435-42.

61. Jung YJ, Lee DG, Cho YW, et al. Effect of intradiscal monopolar pulsed radiofrequency on chronic discogenic back pain diagnosed by pressure-controlled provocative discography: a one year prospective study. Ann Rehabil Med 2012;36:648-56.

62. Rohof O. Intradiscal pulsed radiofrequency application following provocative discography for the management of degenerative disc disease and concordant pain: a pilot study. Pain Pract 2012;12:342-9.

63. Teixeira A, Sluijter ME. Intradiscal high-voltage, longduration pulsed radiofrequency for discogenic pain: a

Cite this article as: Yang S, Chang MC. Efficacy of pulsed radiofrequency in controlling pain caused by spinal disorders: a narrative review. Ann Palliat Med 2020;9(5):3528-3536. doi: 10.21037/ apm-20-298 preliminary report. Pain Med 2006;7:424-8.

64. Chang MC. Effect of Pulsed Radiofrequency Treatment on the Thoracic Medial Branch for Managing Chronic Thoracic Facet Joint Pain Refractory to Medial Branch Block with Local Anesthetics. World Neurosurg 2018;111:e644-8.

65. Atim A, Ergin A, Bilgiç S, Deniz S, et al. Pulsed radiofrequency in the treatment of coccygodynia. Agri 2011;23:1-6.

66. Gopal H, Mc Crory C. Coccygodynia treated by pulsed radio frequency treatment to the Ganglion of Impar: a case series. J Back Musculoskelet Rehabil 2014;27:349-54.

67. Usta B, Gozdemir M, Sert H, et al. Fluoroscopically guided ganglion impar block by pulsed radiofrequency for relieving coccydynia. J Pain Symptom Manage 2010;39:e1-2.

68. Kim MS, Lee DG, Chang MC. Outcome of Transforaminal Epidural Steroid Injection According to Severity of Cervical Foraminal Stenosis. World Neurosurg 2018;110:e398-e403.

69. Kwak S, Chang MC. Management of refractory chronic migraine using ultrasound-guided pulsed radiofrequency of greater occipital nerve: Two case reports. Medicine (Baltimore) 2018;97:e13127.

70. Nathan ST, Fisher BE, Roberts CS. Coccydynia: a review of pathoanatomy, aetiology, treatment and outcome. J Bone Joint Surg Br 2010;92:1622-7. 